University of Nebraska - Lincoln

DigitalCommons@University of Nebraska - Lincoln

Roger Kirby Publications

Research Papers in Physics and Astronomy

May 2000

\title{
Activation volumes in thin film and particulate systems
}

Roger D. Kirby

University of Nebraska-Lincoln, rkirby1@unl.edu

Mingjun $\mathrm{Yu}$

University of Nebraska - Lincoln

David J. Sellmyer

University of Nebraska-Lincoln, dsellmyer@unl.edu

Follow this and additional works at: https://digitalcommons.unl.edu/physics_kirby

Part of the Physics Commons

Kirby, Roger D.; Yu, Mingjun; and Sellmyer, David J., "Activation volumes in thin film and particulate systems" (2000). Roger Kirby Publications. 6.

https://digitalcommons.unl.edu/physics_kirby/6

This Article is brought to you for free and open access by the Research Papers in Physics and Astronomy at DigitalCommons@University of Nebraska - Lincoln. It has been accepted for inclusion in Roger Kirby Publications by an authorized administrator of DigitalCommons@University of Nebraska - Lincoln. 


\title{
Activation volumes in thin film and particulate systems
}

\author{
Roger D. Kirby, ${ }^{\text {a) }}$ Mingjun Yu, and D. J. Sellmyer \\ Department of Physics and Astronomy and Center for Materials Research and Analysis, \\ University of Nebraska, Lincoln, Nebraska 68588-0111
}

\begin{abstract}
We consider magnetization reversal and decay in thin film and particulate magnetic systems where thermally activated processes are important. We present measurements on the activation volumes associated with magnetization reversal in $\mathrm{CoCrPt}$ thin films. We find that the measured activation volume $V^{*}$ goes through a minimum as the film thickness is decreased from 17.5 to $5.5 \mathrm{~nm}$. We have modeled this behavior qualitatively by assuming that the energy barrier to thermally activated reversal varies as $1 / H$, as has been predicted for incoherent nucleation processes and thermally activated domain-wall motion. This model also predicts that $V^{*}$ varies as $T^{2}$ at high temperature in qualitative agreement with measurements on several systems. This behavior is contrasted with that predicted on the basis of coherent rotation of the magnetization in single-domain particles. (C) 2000 American Institute of Physics. [S0021-8979(00)44708-0]
\end{abstract}

Magnetization reversal in most magnetic materials occurs through nucleation of a region of reversed magnetization and/or expansion of the reversed domain by domainwall motion. Both the nucleation and domain-wall expansion processes can be thermally activated, and considerable effort has been expended in recent years to estimate the size of the activation volume $V^{*}$ during reversal. $V^{*}$ is also an important parameter if one is concerned with the time and temperature stability of recorded bits in magnetic thin films. An estimate of $V^{*}$ can be obtained from magnetic viscosity measurements using a variety of approaches, or from the sweep rate dependence of the coercive field. ${ }^{1-3}$ Most often, the values of $V^{*}$ are interpreted in terms of reversal by coherent rotation of the moments in singledomain particles. However, this model cannot explain the observed temperature dependence of $V^{*}$ in many materials or the recently observed minimum in $V^{*}$ as a function of film thickness in Co-based recording media. ${ }^{4,5}$ The present article addresses the issue of physical interpretation of the measured $V^{*}$. We will see that $V^{*}$ is not in general simply related to a physical volume in the material, which means that $V^{*}$ results must be interpreted with considerable care. Further, we will see that assuming that the energy barrier to thermally activated reversal varies as $1 / H$ results in behavior that is qualitatively consistent with experiment in $\mathrm{CoCrPt}$ thin film media.

Let us first consider a simple system of $N_{0}$ identical magnetic particles of volume $V$, saturation magnetization $M_{s}$, and uniaxial anisotropy constant $K_{u}$. Let $E_{B}(H)$ be the magnetic field dependent energy barrier to thermal activation. Then we can write

$$
\frac{d N}{d t}=-f_{0} N \exp \left(-\frac{E_{B}(H)}{k_{B} T}\right),
$$

\footnotetext{
a) Author to whom correspondence should be addressed; electronic mail: rkirby1@unl.edu
}

where $f_{0}$ is an attempt frequency typically taken as $10^{9} \mathrm{~Hz}$. We now assume that $d N / d t$ takes on its maximum value when $H=H_{c}$. Then

$$
\frac{d}{d t}\left(\frac{d N}{d t}\right)=0 \quad \text { at } H=H_{c}
$$

so

$$
\begin{aligned}
0= & -f_{0} \frac{d N}{d t} \exp \left(-\frac{E_{B}\left(H_{c}\right)}{k_{B} T}\right)-f_{0} N \exp \left(-\frac{E_{B}\left(H_{c}\right)}{k_{B} T}\right) \\
& \times\left(-\frac{1}{k_{B} T} \frac{d E_{B}\left(H_{c}\right)}{d t}\right) .
\end{aligned}
$$

After simplification, this leads to

$$
f_{0} \exp \left(-\frac{E_{B}\left(H_{c}\right)}{k_{B} T}\right)+\frac{1}{k_{B} T} \frac{d E_{B}\left(H_{c}\right)}{d t}=0 .
$$

It is often assumed that $E_{B}$ depends linearly on the applied field, so that

$$
E_{B}=E_{0}-M_{s} V H .
$$

Then

$$
\frac{d E_{B}}{d t}=-M_{s} V \dot{H} .
$$

Substituting into Eq. (1) leads to the result

$$
H_{c}=\frac{k_{B} T}{M_{s} V} \ln \left(\frac{M_{s} V}{f_{0} k_{B} T}\right)+\frac{E_{0}}{M_{s} V}+\frac{k_{B} T}{M_{s} V} \ln (\dot{H}),
$$

i.e., the slope of the $H_{c}$ vs $\ln (d H / d t)$ curve can be used to determine the particle volume. A similar result has been derived by Bruno et al. ${ }^{6}$ For the energy barrier given by Eq. (2), we see that the volume $V^{*}$ determined by this method is equal to the particle volume $V$ :

$$
V^{*}=\frac{k_{B} T}{S M_{s}}=V,
$$

where $S$ is the slope of the $H_{c}$ vs $\ln (d H / d t)$ curve. 
The assumption that $E_{B}$ is given by Eq. (2) is not generally true. Consider a system for which the energy barrier has a more complex dependence on $H, E_{B}=E_{B}(H)$ and expand in a Taylor series about $H_{c}$ :

$$
\begin{aligned}
E_{B} & \approx E_{B}\left(H_{c}\right)+\frac{d E_{B}\left(H_{c}\right)}{d H}\left(H-H_{c}\right) \\
& =\left[E_{B}\left(H_{c}\right)-H_{c} \frac{d E_{B}\left(H_{c}\right)}{d H}\right]+\frac{d E_{B}\left(H_{c}\right)}{d H} H \\
& =E_{0}+\frac{d E_{B}\left(H_{c}\right)}{d H} \cdot H .
\end{aligned}
$$

Thus, comparing Eqs. (2) and (5), we see that $d E_{B} / d H$ plays the role of $-M_{s} V$ in determining the slope of the $H_{c}$ vs $\ln (d H / d t)$ curve, a point that has been recognized by Gaunt. ${ }^{7}$ This can have a striking effect on the activation volumes $V^{*}$ determined from magnetic viscosity or sweep-rate dependence of coercivity measurements. For example, if we consider single-domain particles that reverse by coherent rotation, the energy barrier to thermal activation is given by

$$
E_{B}=K_{u} V\left(1-\frac{H}{H_{K}}\right)^{m},
$$

where $H_{K}=2 K_{u} / M_{s}$ is the anisotropy field and $m=2$ for aligned particles and $m \sim 3 / 2$ for randomly oriented particles

$$
\begin{aligned}
\frac{d E_{B}}{d H} & =-\frac{m K_{u} V}{H_{K}}\left(1-\frac{H}{H_{K}}\right)^{m-1} \\
& =-\frac{m M_{s} V}{2}\left(1-\frac{H}{H_{K}}\right)^{m-1} .
\end{aligned}
$$

In this case, we see that

$$
V^{*}\left(H_{c}\right)=\frac{m V}{2}\left(1-\frac{H_{c}}{H_{K}}\right)^{m-1} .
$$

We can carry this a bit further and calculate the temperature dependencies of $H_{c}$ and $V^{*}$. For a given sweep rate (measurement time) we have

$$
\frac{E_{B}\left(H_{c}\right)}{k_{B} T} \approx 25
$$

(depending on $f_{0}$ and $d H / d t$ ).

So we have

$$
\frac{K_{u} V}{k_{B} T}\left(1-\frac{H_{c}}{H_{K}}\right)^{m} \approx 25
$$

or

$$
1-\frac{H_{c}}{H_{K}}=\left(\frac{25 k_{B} T}{K_{u} V}\right)^{1 / m} .
$$

Therefore,

$$
V^{*}\left(H_{c}\right)=V\left(1-\frac{H_{c}}{H_{K}}\right)^{m-1}=V\left(\frac{25 k_{B} T}{K_{u} V}\right)^{(m-1) / m},
$$

where $V^{*} \propto T^{1 / 2}$ for aligned particles; $V^{*} \propto T^{1 / 3}$ for randomly oriented particles. In these cases, the measured activation volume will approach the real particle volume at high tem- perature and will approach zero at low temperature. It should also be noted that numerical calculations using procedures similar to those discussed by Lottis et al. ${ }^{8}$ and by Kirby et $a l .{ }^{9}$ give results in agreement with the above analytical results. This is not surprising given the fact that reversal, on the time scale being considered, occurs only when $H$ is near $H_{c}$.

Now we consider magnetization reversal when the field dependence of the energy barrier is quite different from that of Eq. (2). Egami ${ }^{10}$ considered the problem of thermally activated domain-wall motion in high anisotropy materials and showed that the energy barrier to reversal by domain-wall bubble formation varies approximately as $1 / \mathrm{H}$. A similar result was obtained by Barbara and Uehara. ${ }^{11}$ More recently, Hinzke and Nowak ${ }^{12}$ considered reversal by nucleation in a spherical particle and showed that the energy barrier to reversal varies as $1 / H^{2}$; for a two-dimensional particle one would have a $1 / H$ variation. A common feature in all of these cases is that reversal occurs by incoherent rotation processes, suggesting that a $1 / H$ dependence might be endemic to such processes. In particular, one might speculate that incoherent processes will be especially important in real nanoscaled magnetic materials such as those being used in magnetic recording applications. Materials like $\mathrm{CoCrPt}$ have complex magnetic structures, with impurities, exchange interactions, grain boundaries, columnar growth, etc., with local sites that may serve as nuclei to initiate incoherent reversal processes.

With this discussion in mind, we assume that the energy barrier to thermally activated reversal has the form

$$
E_{B}=A V\left(\frac{1}{H}-\frac{1}{H_{0}}\right),
$$

where $A$ is a constant, $V$ is a physical volume in the magnetic material, and $H_{0}$ represents a nucleation field or a pinning field for domain-wall motion. Then an analysis as in Eqs. (3) - (7) leads to the results

$$
\begin{aligned}
& H_{c}=\frac{A V H_{0}}{1+25 k_{B} T H_{0}}, \\
& V^{*}\left(H_{c}\right)=\frac{\left(A V+25 k_{B} T H_{0}\right)^{2}}{M_{s} A V H_{0}^{2}} .
\end{aligned}
$$

Note that this predicts that the activation volume varies as $T^{2}$ at high temperature. Behavior qualitatively similar to this has been observed in $\mathrm{CoSm}$ thin films ${ }^{13}$ and in $\mathrm{Co} / \mathrm{Au}$ multilayers. ${ }^{14}$

Now let us compare these predictions with behavior observed in CoCrTa. Yu et al. have studied thin films of CoCrTa grown on a $\mathrm{Cr}$ substrate with film thicknesses varying between 5 and $500 \mathrm{~nm}$. ${ }^{4}$ They report columnar growth in this system, with CoCrTa columns roughly in the shape of cylinders of diameter $d=15 \mathrm{~nm}$ and of length equal to the film thickness $t$. We assume that the particle volume $V$ is then $\pi t d^{2} / 4$. Yu et al. also report the very interesting result that $V^{*}$ passes through a minimum with decreasing film thickness. Figure 1 (connected squares) shows their experimental results for $V^{*}$ as a function of film thickness. The solid curve is the prediction of Eq. (8) with $M_{s}$ $=469 \mathrm{emu} / \mathrm{cm}^{3}, T=300 \mathrm{~K}, A=1.99 \times 10^{9} \mathrm{erg} \mathrm{Oe} / \mathrm{cm}^{3}$, and 


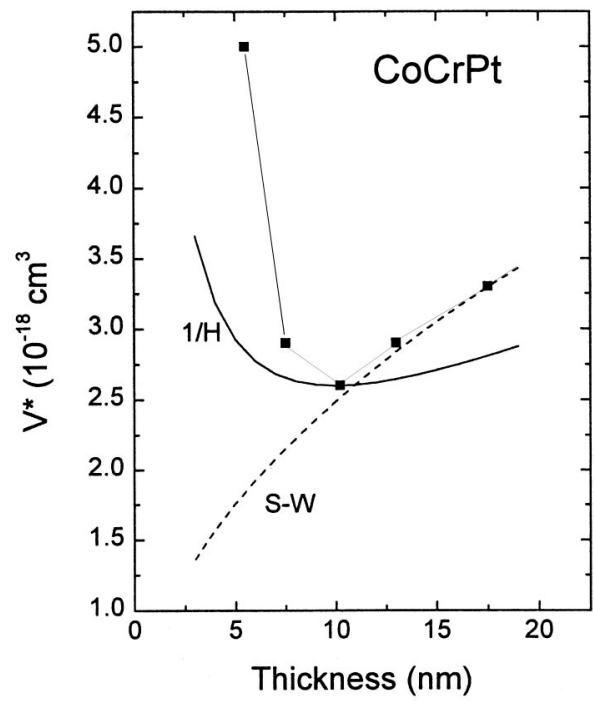

FIG. 1. $V^{*}$ as a function of CoCrPt film thickness (connected squares). The solid line is the prediction of Eq. (8), and the dashed line is the predicted result for coherent rotation in single domain particles [Eq. (7), $m=2$ ].

$H_{0}=3394$ Oe. While this model does predict the observed minimum in $V^{*}$, the agreement is only qualitative. For comparison, the result for reversal by coherent rotation of single domain particles [Eq. (7), $m=2$ ] is shown as a dashed line.

Figure 2 shows the calculated temperature dependence of $V^{*}$ using the same parameters as above for several different film thicknesses. Note in particular that the curves intersect; the small particles in the thinner films reach their high temperature behavior at lower temperatures than do the larger particles in the thicker films. The vertical line at $300 \mathrm{~K}$

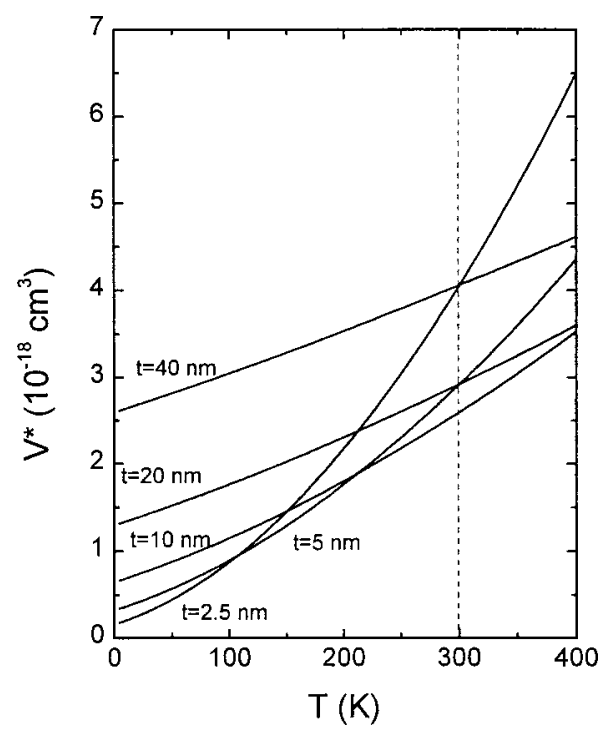

FIG. 2. $V^{*}$ as a function of temperature for several different film thicknesses. The curves were calculated using Eq. (8).

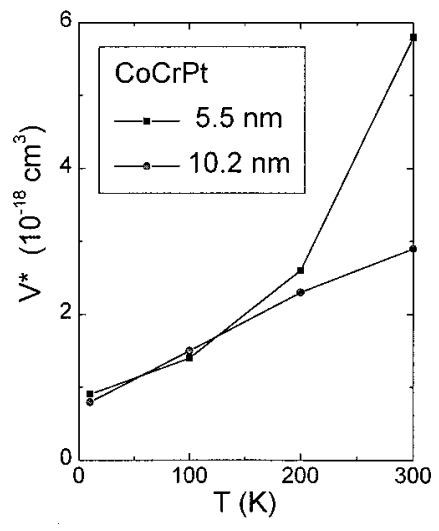

FIG. 3. Temperature dependence of $V^{*}$ for CoCrPt films of two different thicknesses $t$ (filled circles) $t=10.2 \mathrm{~nm}$; (filled squares) $t=5.5 \mathrm{~nm}$.

indicates that $V^{*}$ passes through a minimum as the film thickness decreases.

Figure 3 shows the temperature dependence of $V^{*}$ for CoCrPt films of thickness 5.5 and $10.2 \mathrm{~nm}$. Note that at $300 \mathrm{~K}, V^{*}$ for the $5.5 \mathrm{~nm}$ film is greater than for the $10.2 \mathrm{~nm}$ film, and the two curves seem to coalesce at low temperatures. This behavior is qualitatively similar to the calculated behavior in Fig. 2.

In summary, we have shown that some features of the behavior of the activation volume in $\mathrm{CoCrPt}$ thin films can be qualitatively explained by a simple model which assumes a $1 / H$ dependence of the energy barrier to thermally activated reversal. Perhaps the main conclusion of this work is that the measured activation volumes must be interpreted with some consideration of the field dependence of the energy barrier to thermal activation.

This research was supported by the Army Research Office under Grant No. DAAG55-98-1-0014, the National Science Foundation under Grant No. DMR-9623992, and by the National Storage Industry Consortium.

${ }^{1}$ D. J. Sellmyer, R. D. Kirby, and S. S. Jaswal, in The Magnetism of Amorphous Metals and Alloys, edited by J. A. Fernandez-Baca and W. Y. Ching (World Scientific, Singapore, 1996).

${ }^{2}$ Y. Estrin, P. G. McCormick, and R. Street, J. Phys.: Condens. Matter 1, 4845 (1989)

${ }^{3}$ M. El-Hilo, A. M. de Witte, K. O'Grady, and R. W. Chantrell, J. Magn. Magn. Mater. 117, L307 (1992).

${ }^{4}$ M. Yu, M. Doerner, and D. J. Sellmyer, IEEE Trans. Magn. 34, 1534 (1998).

${ }^{5}$ C. Ross, IEEE Trans. Magn. 34, 282 (1998).

${ }^{6}$ P. Bruno, G. Bayreuther, P. Beauvillain, C. Chappert, G. Lugert, D. Renard, J. P. Renard, and J. Seiden, J. Appl. Phys. 68, 5759 (1990).

${ }^{7}$ P. Gaunt, J. Appl. Phys. 59, 4129 (1986).

${ }^{8}$ D. Lottis, R. White, and E. D. Dahlberg, Phys. Rev. Lett. 67, 362 (1991).

${ }^{9}$ R. D. Kirby, J. X. Shen, R. J. Hardy, and D. J. Sellmyer, Phys. Rev. B 49, 10810 (1994).

${ }^{10}$ T. Egami, Phys. Status Solidi 19, 747 (1973).

${ }^{11}$ B. Barbara and M. Uehara, Inst. Phys. Conf. Ser. 37, 204 (1978).

${ }^{12}$ D. Hinzke and U. Nowak, Phys. Rev. B 58, 265 (1998).

${ }^{13}$ S. S. Malhotra, Z. S. Shan, D. C. Stafford, S. H. Liou, and D. J. Sellmyer, IEEE Trans. Magn. 32, 4019 (1996).

${ }^{14}$ Z. S. Shan, R. D. Kirby, and D. J. Sellmyer (unpublished). 